\title{
Thermal Cycling Simulation in Electronic Packages Using Molecular Dynamic Method
}

\author{
H. B. FAN, Edward, K. L. Chan, Cell K.Y. Wong, and Matthew M.F. YUEN \\ Department of Mechanical Engineering, \\ Hong Kong University of Science and Technology \\ Clear Water Bay, Kowloon, Hong Kong SAR, China \\ mehaibo@ust.hk, Edwardc(@ust.hk cacell@ust.hk meymf@ust.hk \\ Phone: (852) 2358 8814, Fax: (852) 23588357
}

\begin{abstract}
Reliability under thermal cycle conditions is one of the main concerns in electronic packaging design. The cause of this concern arises from the mismatch in coefficient of thermal expansion between the different layers in a package leading to high interfacial stresses induced by thermal loading during fabrication and assembly. If these stresses exceed the limiting value, delamination will occur.

The present study is focused on the reliability of the interfacial failure between conductive polymer (silver epoxy) and gold coated on sample die. The thermal cycling test was conduced with a given thermal profile. The adhesion strength between epoxy and gold coated die subjected to at different thermal cycles was evaluated using the button shear test (BST). A simple molecular model of a bi-material system, which consists of epoxy and gold, was built to evaluate the interfacial energy of the gold-epoxy system. In order to dramatically reduce the computational time, the system was modeled with a limited number of atoms. A preset strain was applied to the model representing a forcing step as the epoxy material was pulled away from the gold substrate. Equilibration was conducted to relax the whole system before the next strain step proceeded. The procedure was repeated using different strains. The interfacial energy induced in the interface under different thermal cycles was evaluated. The trend of the interfacial energy indicates the change of the adhesion strength between epoxy and gold during the thermal cycling test. The simulation results can be benchmarked by the results of BST subjected to the same number of thermal cycles. The simulation result demonstrated that the basic molecular model could be used to predict reliability in the package design.
\end{abstract}

\section{Introduction}

The possibility of using adhesives in interconnect attach application has been given increasing attention. Reliability of dissimilar material systems under thermal cycling conditions is one of the main concerns during the assembly of microelectronic packages. The mismatch in the coefficient of thermal expansion between different layers in packages can generate high interfacial stresses when subjected to thermal loading during fabrication and assembly. These stresses concentrate at the assembly ends and often cause delaminations at the interface.

Finite element analysis has been widely used to model delamination in electronic packages subjected to thermal cycling loading condition. Although the method can provide information to understand the failure mechanism in reliability tests, some phenomena in the tests still cannot be completely explained using the conventional fracture mechanics theory. Moreover, as material properties at the interface are different from those of bulk materials, investigations at molecular level are becoming increasingly important and necessary.

The increasing power of computational methods has offered the potential for molecular dynamic (MD) simulations to predict the mechanical properties of adhesive, which are difficult or laborious to access in the laboratory. The (MD) method was first introduced by Alder and Wainwright in the late 1950's to study the interactions of hard spheres [1]-[2]. Fan, et al [3] demonstrated the application of molecular simulation methods to compute mechanical properties of polymer. Liang, et al [4] investigated the molecular interactions between single-walled carbon nanotube and curing agent molecules using MD simulation. Molecular simulation of the influence of chemical cross links on the shear strength of carbon nanotube/polymer interfaces was conducted by Frankland, et al [5]. MD simulations were also conducted to investigate the molecular interactions between different nano structures and interesting results were presented by other researchers [6-8]. Property trend analysis and simulation of the adhesive formulation effect in the microelectronics packaging industry using $\mathrm{MD}$ simulation were conducted by Iwamoto [9][10], and she accomplished many significant and interesting results in the research of electronic package using MD simulations [11][12]. Although the results from MD simulations could not be directly compared to the experimental measurements, qualitative trends predicted by MD simulation have shown to be in agreement with experimental observations in all the above researches.

In this study, MD simulation was performed to investigate the adhesion under cyclic thermal loading at a the epoxy-gold interface. A reliability model of a bimaterial system, which consists of epoxy and gold coated silicon, was built to evaluate the interfacial energy of the gold-epoxy system. The interfacial energy at different 
cycles was evaluated. The thermal cycling varying trend of the interfacial energy indicated the change of the adhesion strength between epoxy and gold during the thermal cycling test. The thermal cycling test was conducted with a given thermal profile. The adhesion between epoxy and die at different thermal cycles was evaluated using Button Shear Test (BST). The simulation results give a qualitative trend which show to be agreement with the results of BST after the thermal cycling test.

\section{Simulation of the Thermal Cycling Test}

\section{Selection of Proper Force Field}

A main ingredient in MD simulation is the description of forces between atoms as their centres move relative to each other. This description is characterized by a force freld, which can be considered for analytical purposes, to be a collection of energy terms charactering imteractions between atoms of a system. The potential energy, $E$, of a generic force field for an arbitrary geometry of molecules is typically characterized by the following terms:

$$
E=E_{B}+E_{A}+E_{T}+E_{I}+E_{v d w}+E_{Q}
$$

where $E_{B}$ is bond stretching energy, $E_{A}$ is bending energy, $E_{T}$ is torsion energy, $E_{I}$ is inversion energy, $E_{v d w}$ is Van der Waals energy, $E_{Q}$ is electronic energy.

The realism of the computation results hinges on the commensurate choice of the force field. A large number of force fields are available in the literature, optimized to represent different type of physical systems. To represent the bi-material system of a amorphous polymer and metal, there are three most commonly used force fields, constant valence force filed (CVFF), polymer consistent force field (PCCF), and condensectphase optimized molecular potentials for atomistic simulation studies (COMPASS).

The CVFF was the original force field provided in the discover software simulation package and has been augmented on subsequent releases. CVFF was designed for small organic crystals and gas phase structures. The PCFF for polymers and other materials is intended for application to polymers and organic materials. It is useful for polycarbonates, melamine resins, polysaccharides, other polymers, organic and inorganic materials, about 20 metals, as well as for carbohydrates, lipids, and nucleic acids. PCFF can also be used to calculate cohesive energies, mechanical properties, compressibility, heat capacities, and elastic constants. In comparison with other force fields, COMPASS is the first ab initio force field that was parameterized and validated using condensed-phase properties (in addition to various ab initio and empirical data for molecules in isolation). Consequently, this force field enables accurate and simultaneous prediction of structural, conformational, vibrational, and thermo physical properties for a broad range of molecules in isolation and in condensed phases. The advantage of the COMPASS force field is its high accuracy in predicting the molecular properties of polymers, metals and their interfaces. Therefore, COMPASS force field was selected in this study.

\section{The Reliability Model of Silver Epoxy/Silicon System}

When fabricating silver epoxy composites, Diglycidyl Ether of Bisphenol-A (DGEBA) epoxy resin reacted with Methylene Diamine Dianilene (MDA) curing agent. During the curing reaction, the hydrogen atoms in the amine groups of curing agent molecules reacted with the epoxide groups of epoxy reins. Initially, one epoxide group reacted with one curing agent molecules. The resulting molecule can further react with three epoxy molecules at the site of $\mathrm{NH}$ and $\mathrm{NH} 2$ and one curing agent molecule at the site of another epoxide group, as shown in Figure 1.

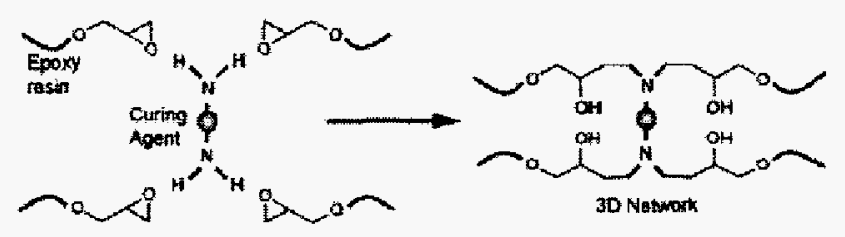

Figure 1: Reaction of a curing agent with four epoxide groups

In the molecular dynamics simulations, a periodic model of the silver epoxy system was used. By applying periodic boundaries to the simulations, the influence of bulk environments could be included, therefore improving the accuracy and realism of a model. Hence, the properties of a molecular level system and a macroscopic system are essentially the same. The bulk amorphous state was built with the polymer chains packed into a unit cell and the cell was repeated in two directions with a confined layer for building the model of silver epoxy and gold coated silicon.

Gold surfaces were cleaved from a crystal gold structure, corresponding to the (001) plane. As the nonbond cut off distance in the force field setting was $9.5 \dot{A}$, the depth of copper surface used in the simulation 
was about $10 \dot{A}$. A layer builder was used to build a sandwich and a large vacuum spacer was positioned at the top of the copper surface in order to avoid interaction across the mirror image in the $\mathrm{z}$ direction in the calculations. The silver epoxy chains were initially placed on the gold coated silicon substrate. The unit cell was extended in the $x$ and $y$ directions to create a rectangular simulation box $\left(12.34 \times 1234 \dot{A}^{2}\right)$, periodic in the plane parallel to the surface, as shown in Figure 2.

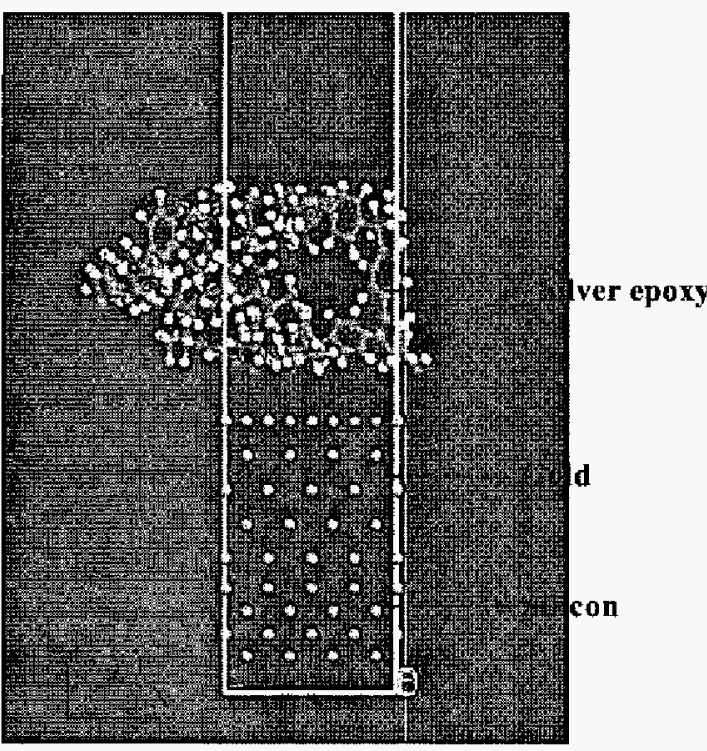

Figure 2: The MD model of the epoxy and gold coated silicon system

The thermal cycling model was constructed using a preset strain target during each simulation step. In a cooling step, a set strain was applied to the model during a forcing step in which the silver epoxy material was pulled away from the gold coated silicon substrate. Equilibration was conducted to relax the whole system at the cooling temperature; while in a heating step, a different strain was applied to the model during a forcing step in which the silver epoxy material was pushed close to the copper substrate. The whole system was then relaxed at the heating temperature. The cooling and heating procedure was repeated using different strains.

\section{Thermal Cycling Test}

A thermal cycling experiment was conducted to benchmark the above $\mathrm{MD}$ simulation result. The samples were fabricated with conductive adhesive on the silicon substrate using a PDMS mould. The samples were cured inside a conventional oven at $80^{\circ} \mathrm{C}$ for $1 \frac{1 / 2}{2}$ hours.

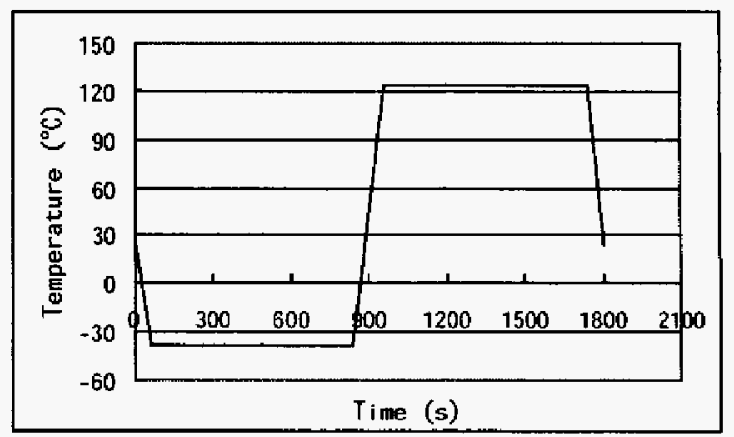

Figure 3: The profile of the thermal cycles

After the cure of the samples, they were put inside the Vosch thermal chamber with temperature profile as shown in Figure 3. The thermal loading starts with a heating from the stress free temperature of $25^{\circ} \mathrm{C}$, followed by a cooling down to $-40^{\circ} \mathrm{C}$ and then heating up to $125^{\circ} \mathrm{C}$.

Samples were removed from the thermal chamber at the prescribed number of thermal cycles. Shear tests were performed using the Dage 4000 shear tester with a DS100 load cell The button shear test specimen was fixed on the mounting jig attached onto the shear tester platform: A special testing jig was designed and made for clamping the sample to cater for testing. A horizontal load with a loading rate of $85 \mu \mathrm{m} / \mathrm{s}$ was applied to the button surface with a controlled loading height, as shown in Figure 4. The breaking load of the specimen with interfacial fracture was recorded.

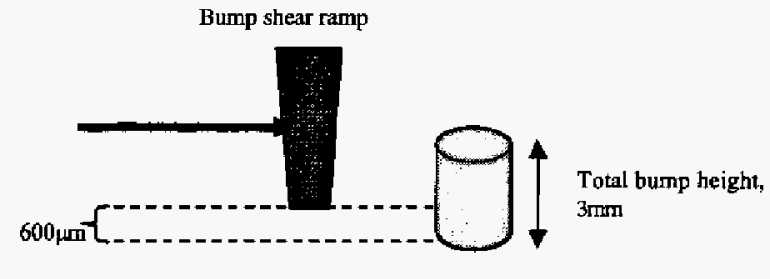

Figure 4: The Schematic drawing of the button shear test

\section{Results and Discussion}

The bonding strength between the epoxy and the copper tip could be evaluated by examining the potential energy. The total potential energy was represented by the superposition of valence and non-bond interactions. The 
valence terms consist of bond stretch, bond angle bending, and dihedral angle torsion terms, while nonbond interactions consist of Van der Waals and electrostatic terms. The interaction between the epoxy and the copper tip is a non-bond interaction. Hence the interfacial bonding energy came from the dectrostatic and van der Waals forces in the molecular system. Generally, the interaction energy was estimated from the energy difference, $\Delta E$, between the total energy of the whole system and the sum of the energies of individual molecules as follows:

$$
\Delta E=E_{t o t a l}-\left(E_{E p o x y}+E_{S i+A u}\right)
$$

where $E_{\text {fotal }}$ is the total energy of the whole system, $E_{\text {Epoxy }}$ is the energy of the epoxy without the gold coated silicon substrate, $E_{S i+A u}$ is the energy of the gold coated silicon substrate without the epoxy. The interfacial bonding energy, $\gamma$, was evaluated using the interfacial energy $\Delta E$ and the contact area $A$ between the epoxy and gold coated silicon substrate:

$$
\gamma=\Delta E / A
$$

Based on above equations, the bonding energies between the silver epoxy and gold coated silicon substrate were plotted against thermal cycles, as shown in Figure 5. It can be seen that the bonding energy for the thermal cycling test generally decreased with the increase of the thermal cycle. The higher bonding energy means the higher the force needed to separate the epoxy from the gold coated silicon substrate. The reason for the decrease of the bonding energy is that large voids forming at the interface during the thermal cycling test reduce the adhesion between the epoxy and silicon substrate. During the thermal cycling test, small voids forms at the interface due to the interfacial stresses resulted from the CTE mismatch of epoxy and gold coated silicon substrate. And the stresses pull epoxy away from the silicon substrate step by step during the thermal cycling. Therefore, aggregation of voids form large cracks at the interface which make the interface weaker and weaker during thermal cycling test This simulation result explained the phenomena in the thermal cycling test on the molecular level.

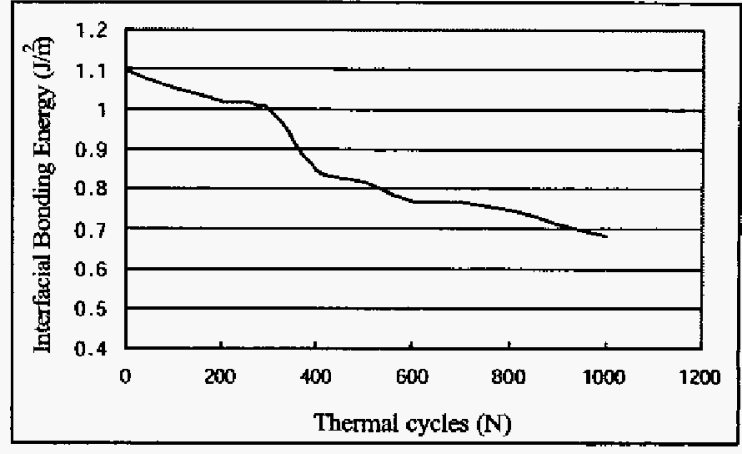

Figure 5: The bonding energy against thermal cycles

The experimental data obtained from the button shear test with different thermal cycles were shown in Figure 6 . The trend showed a decrease in adhesion with the thermal cycles. The experimental result provided a same trend as that in the MD simulation as shown in Figure 5 .

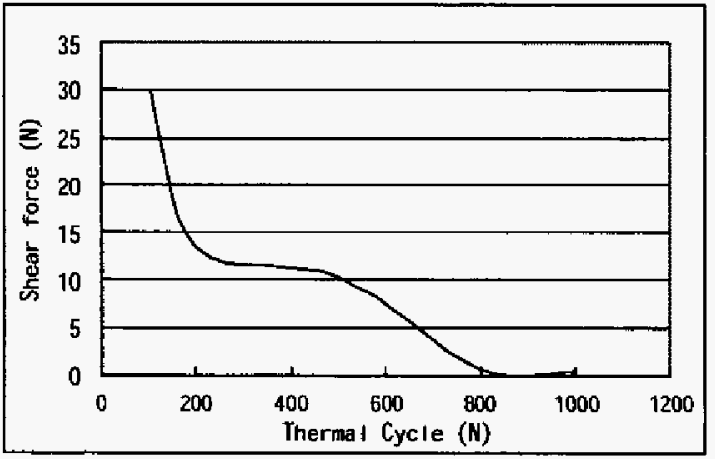

Figure 6: Failure force obtained from different numbers of thermal cycles

This MD result indicates that the adhesion strength of the epoxy and gold coated silicon is more significantly affected by the thermal cycling test. This is consistent with the observation in the thermal cycling test result. The results from $\mathrm{MD}$ simulations demonstrated interactions between epoxy and silicon substrate during the thermal cycling test. The basic trend of adhesion followed an interaction energy trend. Higher the interaction energy gives, higher the adhesion. Although the values from $\mathrm{MD}$ simulations could not be directly compared to the experimental measurements, qualitative trends predicted by $\mathrm{MD}$ simulation have shown to be in agreement with experimental observations. And the simulation results can be used as a guide for further researches. 


\section{Conclasions}

The paper is focused on reliability prediction using a computational model during the thermal cycling test. The present molecular model helped to explain results in the the rmal cycling test, and it indicates a basic relation between the molecular performance and the macroscopic performance. The model revealed that the bonding energy for the thermal cycling test generally decreased with the increase of the thermal cycle. Higher bonding energy gives, higher the adhesion between the epoxy and the gold coated silicon substrate. The thermal cycling test was conducted and the adhesion for different thermal cycles was measured using button shear test. And a qualitative trend predicted by MD simulation has shown to be in agreement with experimental observations.

\section{Acknowledgments}

The project was supported by the Research Grant Council project (HKUST6046/01E), The authors would like to thank Dr. Iwamoto for her suggestion on the study.

\section{References}

[1] B. J. Alder, T. E. Wainwright, "Phase Trasition for a Hard Sphere System, Journal of Chemical Physics, Vol. 27, pp. 1208-1211, 1957.

[2] B. J. Alder, T. E. Wainwright, "Studies in Molecular Dynamics. I. General Method," Journal of Chemical Physics, Vol. 31, pp. 459-466, 1959.

[3] C. F. Fan, S. L. Hsu, "Application of the Molecular Simulation Technique to Characterize the Structure and Properties of an Aromatic Polysulfone System 2. Mechnaical and Thermal Properties," Macromoleculars 23, 226. 1992.

[4] Z.Y. Liang, J. Gou, C. Zhang, B. Wang, L. Kramer, and, "Investigation of Molecular Interactions between $(10,10)$ Single -walled Nanotube and Epon 962 Resin/DETDA Curing Agent Molecules," Mterials Science and Engineering A.365, pp.228-234, 2004.

[5] S. J. V. Frankland, A. Caglar, D. W. Brenner, and M. Griebel, " Molecular Simulation of the Influence of Chemical Cross-links on the Shear Strength of Carbon Nanotube -polymer Interfaces," J. Phys. Chem. B, vol. 106, pp. 3046-3048, 2002.

[6] G. Tanaka, L. A. Goettler, "Predicting the Bonding Energy for Nylon 6,6/Clay nanocomposites by Molecular Modelling," Polymer, Vol.43, pp: 541$553,2002$.

[7] I. Yarovsky, E. Evans, "Computer Simulation of Structure and Properties of Crosslinked Polymers:
Application to Epoxy Resin," Polymer, Vol. 43, pp. 963-969, 2003.

[8] A. Robert, and J. Latour, "Molecular Modelling of Biomaterial Surfaces," Current Opinion in Solid State and Materials Science, Vol. 4, pp. 413-417, 1999.

[9] N. Iwamoto, J. Pedigo, "Property Trend Analysis and Simulations of Adhesive Formulation Effects in the Microelectronics Packaging Industry Using Molecular Modelling," 48th Electron. Comp. Tech. Conf., USA pp. 1241-1246, 1998.

[10]N. Iwamoto, "Applying Polymer Process Studies Using Molecular Modelling," Adhesive Joining and Coating Technology in Electronics Manufacturing, 4th International Conference, pp. 182-187, 2000.

[11]N. Iwamoto, 'Advancing Materials Using Interfacial Process and Reliability Simulations on The Molecular Level," Advanced Packaging Materials: Processes, Properties and Interfaces, pp. 14-17, 2000.

[12]N. Iwanoto, "Advancing Polymer Process Understanding in package and Borard Applications through Molecular Modelling," 50 th Electron. Comp. Tech. Conf., USA, pp. 1354-1359, 2000. 